\title{
Analysis of Panoramic Propagation Patterns Mapped From Patients With Persistent Atrial Fibrillation
}

\author{
Nolwenn Tan ${ }^{1,2}$, Elizabeth Cheng ${ }^{1}$, Martyn P. Nash ${ }^{1}$, Martin K. Stiles ${ }^{3}$, Stephan Willems ${ }^{4}$, Pawel \\ Kuklik $^{4}$, Jichao Zhao ${ }^{1}$ \\ ${ }^{1}$ Auckland Bioengineering Institute, University of Auckland, Auckland, New Zealand \\ ${ }^{2}$ IHU-LIRYC, Université de Bordeaux, Bordeaux, France \\ ${ }^{3}$ Waikato Hospital, Hamilton, New Zealand \\ ${ }^{4}$ Department of Cardiology, Electrophysiology, University Medical Center Hamburg-Eppendorf, \\ Hamburg, Germany
}

\begin{abstract}
The purpose of this study is to analyze the electrical propagation patterns from patients with persistent atrial fibrillation (AF). Unipolar electrograms ( 1 minute) were recorded sequentially in atrial chambers from 5 patients with persistent AF prior to ablation using a 64-electrode basket catheter. Raw unipolar signals were processed using a suite of signal processing approaches including: 1) A Savitzky-Golay filter, 2) a standard QRS subtraction and 3) a local variability approach which uses a local integral to enhance the signals. Recurring patterns of electrode activation and AF drivers were then identified by using activation time maps and dominant frequency analysis, and by screening through the entire isopotential movies of each basket recording. Localized sources in the form of either localized reentries $(N=1)$ or focal impulses $(N=4)$ were observed in all of the patients studied. Interestingly, all localized sources were found in the left atrium (LA) near the pulmonary veins with the exception of 1 patient where an additional focal impulse was also detected in the right atrium (RA). Disorganized activity was observed in the $R A$ of 3 patients, though their respective LAs were driven by localized sources. These results suggest that either localized sources in the LA or disorganized activity in the RA or both of these mechanisms may be the predominant triggers of persistent $A F$.
\end{abstract}

\section{Introduction}

Atrial fibrillation is the most prevalent type of cardiac arrhythmia. Several treatments have been developed to treat this arrhythmia over the years. For example, pulmonary vein $(\mathrm{PV})$ isolation through ablation is well known to treat paroxysmal AF [1]. However, currently there is no effective treatment for persistent $\mathrm{AF}$, the more refractory forms of AF. The debate whether AF is sustained by multiwavelets self-replicating across the atria chambers or maintained by localized sources is still ongoing $[2,3]$.

The multiwavelet hypothesis implies that $\mathrm{AF}$ is driven by multiple random irregular waves [2]. Conversely, the localized sources hypothesis suggests that electrical activity in AF is sustained by dominant regions of organized activity in the form of reentries or focal impulses [4-6].

In order to provide critical insights with respect to the mechanisms underlying AF, this study aims to analyze patient specific AF activities independently. Ideally, an improved understanding of the underlying mechanisms of $\mathrm{AF}$ will lead to the development of more precise and targeted treatment strategies for patients with AF.

\section{Materials and method}

\subsection{Materials}

A 64-electrode basket catheter was introduced sequentially in both atrial chambers (except patient \#5) for simultaneous multi-site recording of the atrial activity (Figure 1). One-minute raw unipolar electrograms were recorded at a $2 \mathrm{kHz}$ sampling frequency. Electroanatomical data including atrial chamber geometry and relative electrode positional data were also simultaneously collected using the EnSite Velocity cardiac mapping system (St Jude medical). The recorded EnSite data was exported and post-processed using Matlab.

\subsection{Definitions}

Propagation patterns were categorized into 3 main morphologies based on previous studies [2]: reentries, focal impulses and disorganized activity. Reentry was defined as the existence of a "repetitive circus movement" that occurs at least once per second throughout the recording [2]. A focal impulse was defined as a site for 
A

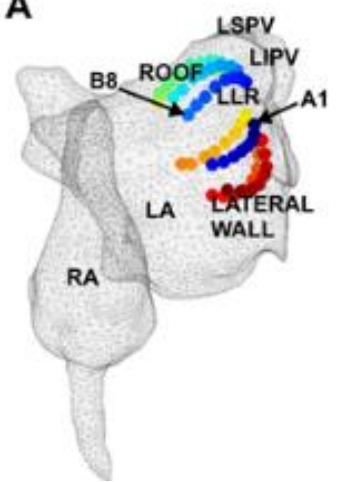

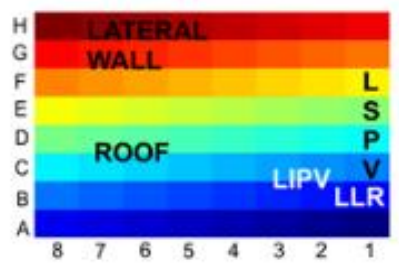

B

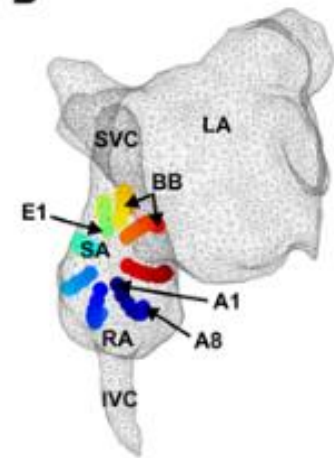

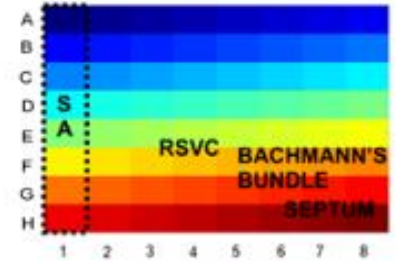

Figure 1. Representation of the 3D atrial geometry showing the basket inside the left $\mathbf{A}$ ) and right $\mathbf{B}$ ) atria. The color here is used to distinguish the 64 electrodes. R/L A: Right/Left Atrium, R S/I VC: Right Superior/Inferior Vena Cava, SA: Sino atrial node, BB: Bachmann's Bundle, L S/I PV: Left Superior/Inferior Pulmonary Vein, LLR: Left Lateral Ridge.

which the activation occurs earlier than all its neighbors and spreads in multiple directions [6]. Propagation patterns that did not fulfill any of these criteria were considered as disorganized activity.

\subsection{Methods}

A Savitzky-Golay filter [7] was applied to the atrial electrograms using a polynomial regression to remove high-frequency noise. A QRS subtraction method [8] was used to cancel far-field ventricular signals from the unipolar electrograms. The unipolar signals were then converted to bipolar to further remove far-field noise. All signals were individually examined where any faulty signals were identified and excluded from further analysis.

The local variability approach described by Ganesan et al. [9] was used to enhance the atrial activity for characterization of the propagation patterns. The local variability $\mathrm{u}\left(t_{k}\right)$ was constructed by computing the local integral of a fixed size sliding window that slides over the recorded signal using the following formula:

$$
u\left(t_{k}\right)=\sum_{m=k-\frac{T}{2 \Delta t}}^{k+\frac{T}{2 \Delta t}}\left|\frac{v\left(t_{m}\right)-v\left(t_{m-1}\right)}{\Delta t}\right|(1)
$$

Here, $\mathrm{T}$ is the size of the sliding window and $\mathrm{v}\left(t_{k}\right)$ is the bipolar signal. The value of $\mathrm{u}\left(t_{k}\right)$ is small during periods of low electrical activity and increases when the sliding window begins to overlap with deflections in the bipolar signal thereby enhancing atrial activity. The dominant frequency was computed using a fast Fourier method. Recurring patterns of activation were then manually identified by using activation time maps, dominant frequency analysis and by screening through the entire isopotential movie.

\section{Results}

\subsection{Reentry}

Reentries were identified in the left atrium (LA) of Patient \#1. Stable and repetitive reentries were detected throughout the entire length of the recording where a consistent pattern was observed across all cycles. Activation circulated around the two left PVs via the posterior LA, atrial roof, atrial ridge and left atrial lateral wall with an average cycle length (CL) of $200 \pm 20 \mathrm{~ms}$ (Figure 2.A). Figure 3.A shows the propagation pattern of the reentries for five cycles (from 1030 to $2060 \mathrm{~ms}$ ). The average frequency of this atrial activity was $4.7 \mathrm{~Hz}$ (Figure 3.B). The core of the reentries was situated in the left PVs.

\subsection{Disorganized activity}

In 3 out the 5 patients studied, disorganized activity was observed in the right atrium (RA). The RA wave propagation in these patients was complex and fibrillatory. Figure 2.B shows the disorganized activity observed in the RA of Patient \#3. The blank spaces represent unreliable signals that were excluded from analysis. Random, irregular wavelets were observed in this patient. This particular activation pattern shows two horizontal waves propagating through the Bachmann's bundle from opposite directions. They collided and were blocked for further propagation. It was not possible to determine the mean cycle length of the propagation pattern as the pattern was entirely anarchical. Similar patterns of chaos and disorganization were also detected in the RA of patients \#1 and \#4. 
A

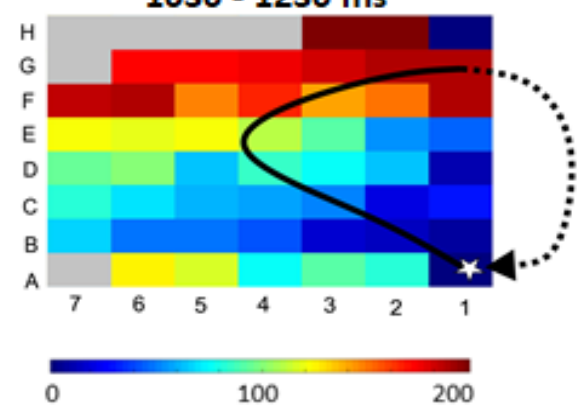

B

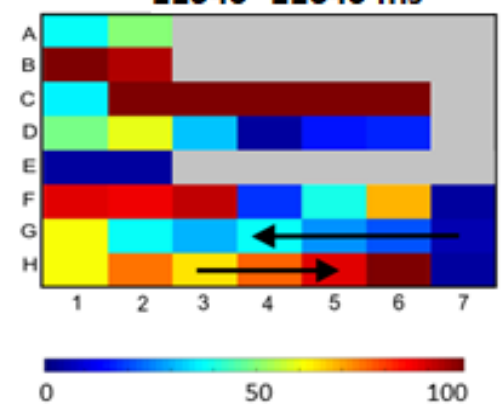

C $\quad 2480-2530 \mathrm{~ms}$

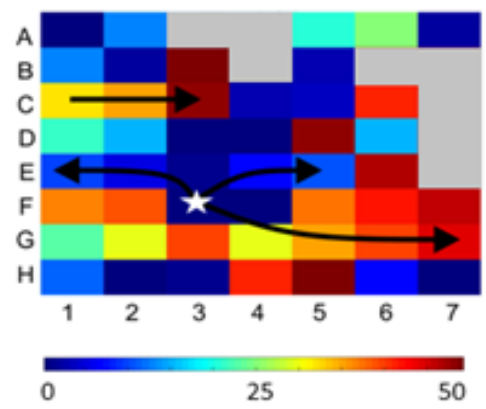

Figure 2. Different activation patterns from 3 patients with persistent atrial fibrillation (AF). A) Left atrial reentry with a mean cycle length (CL) of $200 \pm 20 \mathrm{~ms}$. B) Right atrial disorganized activity. C) Right atrial focal impulses with a mean CL of $150 \pm 20 \mathrm{~ms}$. White spark indicates ectopic foci.

\subsection{Focal impulses}

Focal impulses were observed in the LA of 3 patients and in both atria of 1 patient. The propagation pattern presented in Figure 2.C shows focal impulse located in the RA of patient \#2 near the SVC and the septum. The earliest activation (indicated by the white spark on the activation time map) originated from the electrode F3 and spread radially but non-uniformly towards the rest of the atrium. The focal impulse was observed throughout the entire recording. A similar propagation pattern was observed in the LA of patients \#2, 3, 4 and 5 near the roof and the left superior PVs.

\section{A Bipolar electrograms from 1030 to $2060 \mathrm{~ms}$}

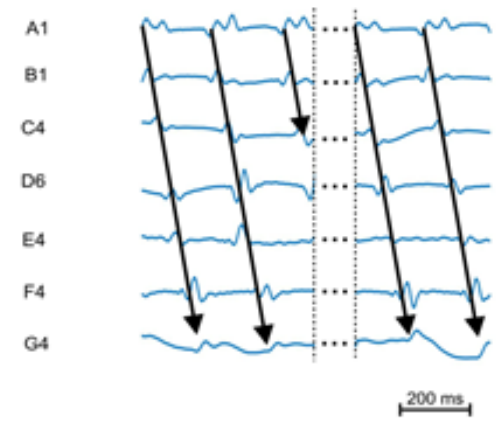

B LA frequency bar chart

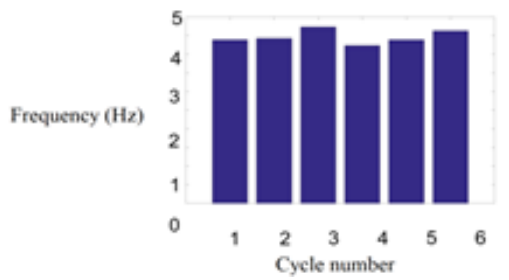

Figure 3. A) Reentries propagation pattern (black arrows) on bipolar signals. Dashed lines: QRS subtraction. B) LA frequency bar chart divided into 6 segments of $10 \mathrm{~s}$.

\section{Discussion}

\subsection{Observation of localized sources}

Although the debate about the mechanisms underlying persistent $\mathrm{AF}$ is still ongoing, sustained localized sources in the form of reentries or focal impulses have been consistently observed in this study. This is in line with findings from several studies $[3,5,6,10,11]$ which suggest that localized sources may play a predominant role in maintaining AF.

\subsection{Disorganized activity in RA}

Disorganized activity was observed in the RAs of patients \#1, 3 and 4 (Figure 4). These observations can either be explained by the multiwavelet hypothesis, suggesting that electrical activity is simply random and disorganized in the RAs of these patients [2], or by the presence of stable localized sources, observed in the contralateral atrium of the same patients, changing into more disorganized activity once passing through the septum.

\subsection{Study limitations}

This study was performed on a small group of patients $(\mathrm{N}=5)$ which is insufficient to represent the true population of patients with persistent AF. Further analysis into a larger database is currently underway. Poor coverage of the atrial surface has limited the understanding of how electrical activity is spread from the localized sources. Poor positioning of the basket catheter also introduced a lot of noise that was difficult to filter out and may lead to the existence of undetected sources. Nevertheless, despite these limitations, we were still able to identify clear patterns of localized activation in all the patients studied. Moreover, the use of the 64-pole basket catheter is a 


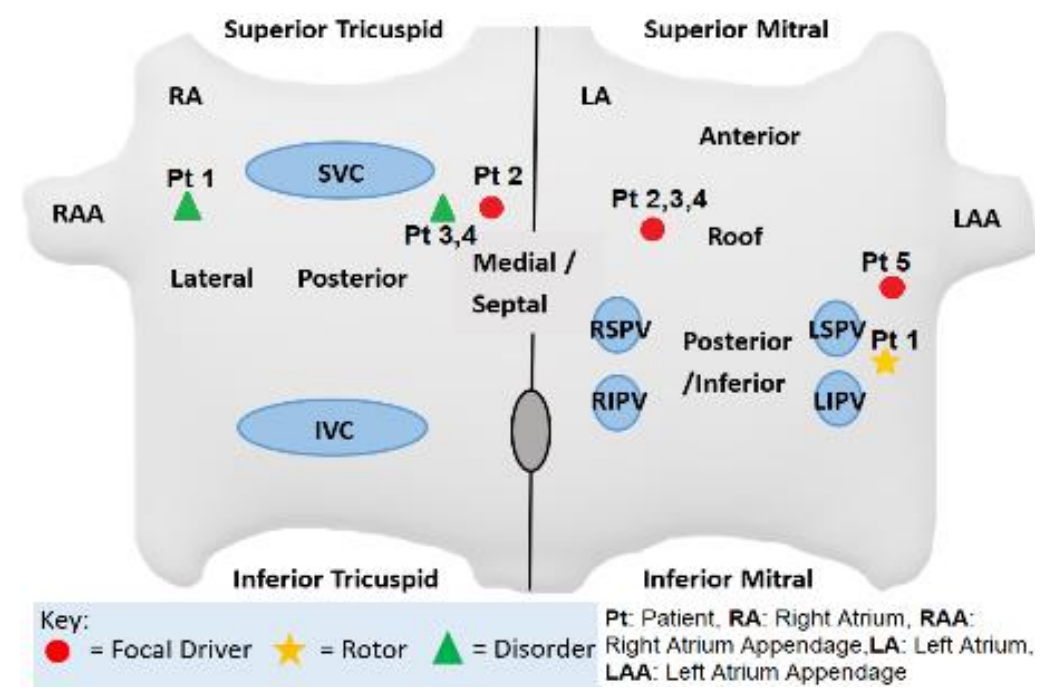

Figure 4. Scheme of the detected activation pattern in both atrial chambers for each patient with persistent atrial fibrillation (N=5). S/I VC: Superior/ Inferior Vena Cava, R/L S/I PV: Right/Left Superior/Inferior Pulmonary Vein

significant parameter to focus on since the electrical propagation pattern might differ depending on the mapping system used.

\section{Conclusion}

The mechanisms sustaining persistent AF are still unclear and the subject of intense debate. In this study, using the 64-pole basket catheter localized sources were mostly observed in the LA and disorganized activity was detected only in the RA. The majority of localized sources are situated near the LSPVs and near the roof which seem to be the major sites of origin of these localized sources. Further analysis will be performed to determine the predominant triggers of persistant AF.

\section{Acknowledgements}

This study was supported by the Health Research Council of New Zealand.

\section{References}

[1] Haissaguerre $\mathrm{M}$ et al. Spontaneous initiation of atrial fibrillation by ectopic beats originating in the pulmonary veins. New England Journal of Medicine 1998; 339(10):659-666.

[2] Lee $\mathrm{G}$ et al. Epicardial wave mapping in human long-lasting persistent atrial fibrillation: transient rotational circuits, complex wavefronts, and disorganized activity. European Heart Journal 2013; 35(2):86-97.

[3] Hansen $\mathrm{BH}$ et al.. Fibrosis and atrial fibrillation: computerized and optical mapping; a view into the human atria at submillimeter resolution. JACC Clinical Electrophysiology 2017; 3(6):531-46.

[4] Kuklik P et al. Identification of rotors during human atrial fibrillation using contact mapping and phase singularity detection: Technical Considerations. IEEE Transactions on Biomedical Engineering 2016; 64(2):310-318.

[5] Haissaguerre $M$ et al. Driver domains in persistent atrial fibrillation. Circulation 2014; 130:530-538.

[6] Hansen BJ et al. Atrial fibrillation driven by microanatomic intramural reentry revealed by simultaneous sub-epicardial and sub-endocardial optical mapping in explanted human hearts. European Heart Journal 2015; 36(35):2390-401.

[7] Savitzky A and Golay MJ. Smoothing and differentiation of data by simplified least squares procedures. Analytical Chemistry 1964; 36(8):1627-1639.

[8] Zhao J et al.. Progressive modification of rotors in persistent atrial fibrillation by stepwise linear ablation. Heart Rhythm Case Reports 2015;1:22-26.

[9] Ganesan A et al. Bipolar electrogram shannon entropy at sites of rotational activation: implications for ablation of atrial fibrillation. Circulation: Arrhythmia and Electrophysiology 2012; 6(1):48-57.

[10] Narayan SM et al. Treatment of atrial fibrillation by the ablation of localized sources: the conventional ablation for atrial fibrillation with or without focal impulse and rotor modulation (CONFIRM) Trial. JACC 2012; 60(7):628-636

[11] Zhao J et al.. 3D integrated functional, structural, and computational mapping to define the structural "fingerprints" of heart-specific atrial fibrillation drivers in human heart ex-vivo. Journal of the American Heart Association 2017; 6:e05922.

Address for correspondence.

Dr. Jichao Zhao

Auckland Bioengineering Institute

University of Auckland, New Zealand

E-mail: j.zhao@auckland.ac.nz 